\title{
Research on home patrol robot
}

\author{
Gai Kerong $^{1, a}$, Niu xiaotie ${ }^{1, b}$ and Zeng jinxiang ${ }^{1, c}$ \\ ${ }^{1}$ Beijing Polytechnic College, No.368 Shimen Road, Shijingshan District Beijing 100042,China \\ akkr0706@126.com, ${ }^{\mathrm{b}}$ niuxit@163.com, ${ }^{\mathrm{c}}$ jinsam520@126.com
}

Keywords: robot home patrol remote monitoring

Abstract. To cater for the demand for home patrol robots, the paper discusses a sort of automatic charging robot which is based on ARMII kernel and wireless embedded S3C6410 processor and realizes the functions of online control via smart terminals through transplanting the Android operation system, home security monitoring through equipping relevant sensors, home entertainment and network assistance. The R\&D prototype and field test demonstrate the system has good stability and flexibility in automatic obstacle avoidance, remote monitoring, home security monitoring and network assistance.

\section{Introduction}

With a growing trend of social aging, accelerated pace of life and the increase of "empty-nest" elderly people, the demand for home patrol and remote monitoring robots is increasingly high. Developed based on computing processing, artificial intelligence, sensing technology and Internet technology, the home service robot with functions of elderly assistance and caring, housework, education and entertainment will enter families at an extra high speed. The paper discusses prototype of the home patrol robot, which has the functions of automatic obstacle avoidance indoors, remote monitoring, testing home functions through sensors, real time transmission, saving and monitoring of images, and remote $\mathrm{A} / \mathrm{V}$ interaction at smart terminals, voice inquiry assistance and entertainment, to explore the further technical development of service robots.

\section{The general design plan}

The home patrol robot consists of the chassis motion and test module, home security test alarm module, network-based remote monitoring module and home entertainment assistance module. The design principle is real time, multiple functions and high stability. The structure is illustrated in Figure 1.

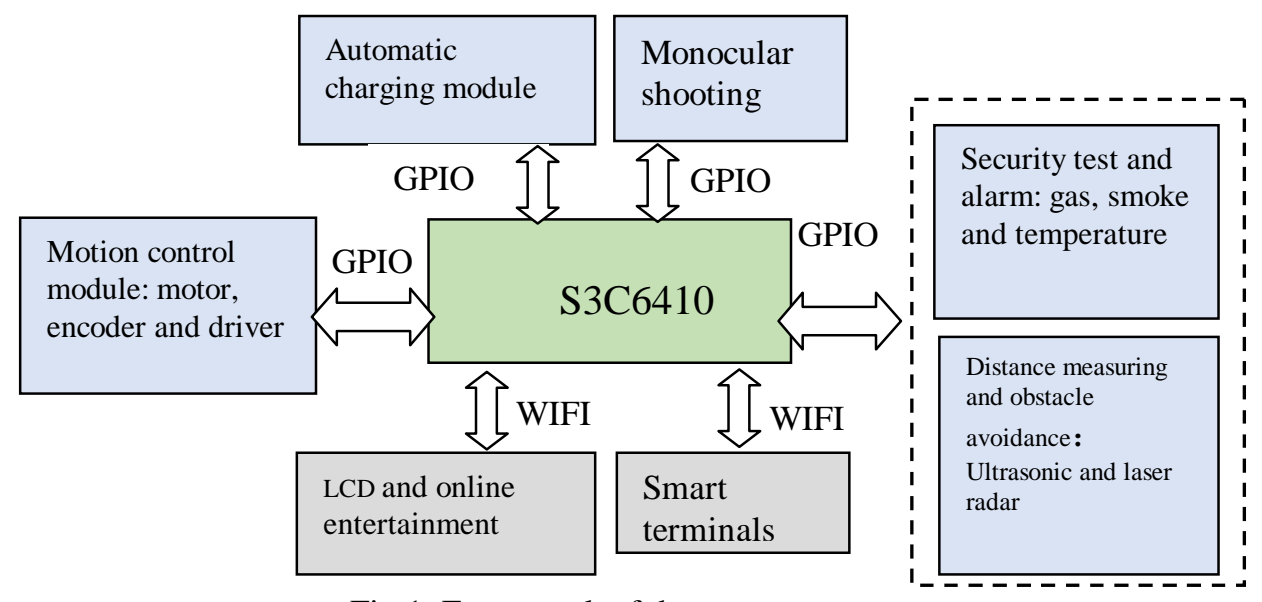

Fig.1 Framework of the system

Based on the ARM11 as its kernel ${ }^{[1]}$, the wireless embedded processor S3C6410, by means of GPIO, collects and processes the image information, distance information measured by ultrasonic and laser radar, signals of gas, temperature and smoke as well as information on battery charge, by means of PWM and the universal IO interface, realized the alarm testing for chassis motor driver control, thus controlling the obstacle avoidance patrol and test of the smart, wireless, patrol robot. 
The prototype development is based on an Android smart phone which is linked with miniPCIe 3G-equipped S3C6410 via a wireless router. The instructions are obtained through HTTP, and the captured image video streams are fed back in wireless mode. The video streams received are decoded and displayed on the mobile phone in real time. The mobile phone sends motion instructions to the S3C6410-based master control system through the TCP-based Socket communication. S3C6410 processes the instructions received and completes the control of the patrol robot in a multithreading mode. A touchscreen LCD can have the functions of home entertainment and network assistance through connection via a wireless router.

\section{The design of three- wheeled omnidirectional chassis}

The aisle in the home environment is normally as wide as allowing one person to pass through and some furniture are relocated from time to time. Therefore the environment is normally complicated. In such characteristics, the robot chassis employs the combination of three- omnidirectional wheels, a servo motor and a photoelectric encoder to allow it moving in all directions on a planar surface ${ }^{[2][3]}$. The assembly of the omnidirectional wheels are illustrated in Fig. 2and Fig.3. The crossing point of the axes of the three wheels, forming a $120^{\circ}$ angle with each other in installation, coincides with the robot's center of gravity, and the slave wheels are perpendicular to the master wheels ${ }^{[4][5]}$ to achieve the optimum driving force .

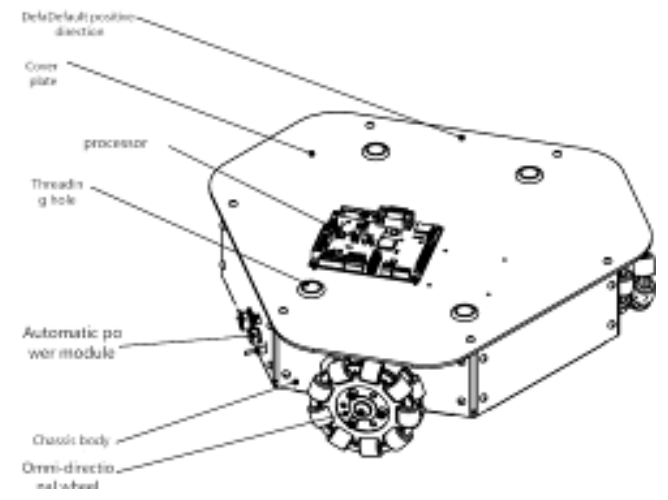

Fig.2 The assembly model for chassis

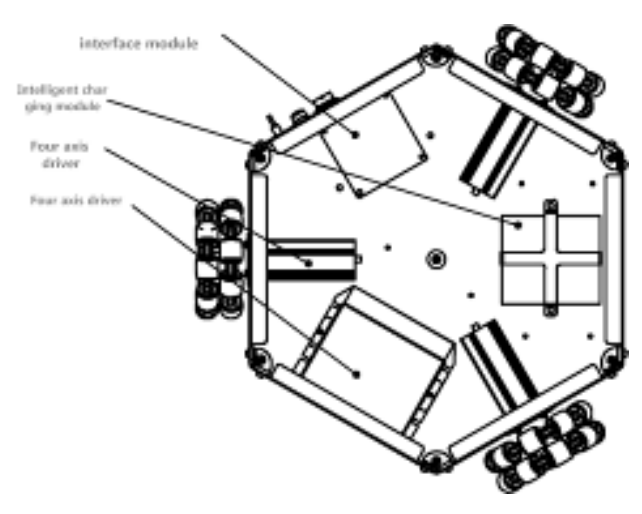

Fig. 3 offers the definition of the chassis motion

Figure 4 offers the definition of the chassis motion coordinate system:of that, $X_{1} O_{1} Y_{1}$ is the overall coordinate system of the robot, ; $\mathrm{X}_{2} \mathrm{O}_{2} \mathrm{Y}_{2}$ is the body-fixed coordinate system of the robot, and the forward direction of the $\mathrm{x}$-axis is the front of the robot and keeps relatively static when the robot is in motion; $\theta$ is the inclination between the overall coordinate system and the body-fixed coordinate

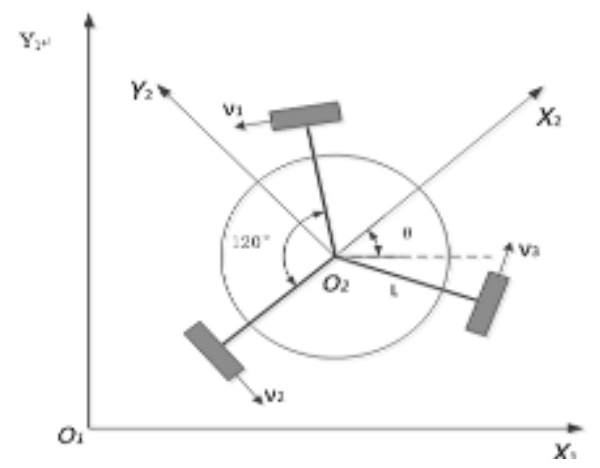

$$
\left[\begin{array}{l}
v_{1} \\
v_{2} \\
v_{3}
\end{array}\right]=\left[\begin{array}{ccc}
-\sin \left(\frac{\pi}{3}+\theta\right) & \cos \left(\frac{\pi}{3}+\theta\right) & L \\
\sin \theta & -\cos \theta & L \\
\sin \left(\frac{\pi}{3}-\theta\right) & \cos \left(\frac{\pi}{3}-\theta\right) & L
\end{array}\right]\left[\begin{array}{c}
\alpha \\
\alpha \\
\theta
\end{array}\right]
$$

Fig.4 the definition of the chassis motion coordinate system 
system; $v_{1}, v_{2}, \quad v_{3}$ are the angular velocity of rotation of each omnidirectional wheel; $L$ is the distance between the center of the omnidirectional wheels and the chassis center. The kinetics equation of the three-wheel omnidirectional chassis is indicated in Formulas 1. The robot's displacement and attitude formulas are Formulas 2. With the kinetic equations, we can work out the attitude increment of the robot chassis and accumulate the increment to finally get the real attitude of the robot. In this system the calculation frequency is $1000 \mathrm{~Hz}$. When the calculation interval is small enough, the error can be controlled to the engineering permissible range.

$$
\left[\begin{array}{l}
\propto \\
\theta
\end{array}\right]=\left[\begin{array}{ccc}
-\sin \left(\frac{\pi}{3}+\theta\right) & \cos \left(\frac{\pi}{3}+\theta\right) & L \\
\sin \theta & -\cos \theta & L \\
\sin \left(\frac{\pi}{3}-\theta\right) & \cos \left(\frac{\pi}{3}-\theta\right) & L
\end{array}\right]^{-1}\left[\begin{array}{l}
\phi \\
\phi \\
\phi \\
\psi
\end{array}\right]
$$

\section{The design of control system}

Wireless video acquisition processor The module combing ZTE AC200 miniPCIe 3G module with the developed 3G dial software can plug and play on the developed board, dial to connect the netwark or send short messages. The module supports EVDO/CDMA with the frequency of CDMA800MHz and a transmission rate of $3.1 \mathrm{M} / 1.8 \mathrm{Mbps}$. In case of detecting abnormal signals of gas or temperature, the robot can send real time short messages to the mobile phone of the designated contact person.

The design of motion control processor S3C6410 is a development platform based on SAMSUNG 16/32-bit RSIC microprocessors. S3C6410X, a universal processor based on the ARM1176JZF-S kernel for handset and mobile terminals, offers optimized hardware performance for $2.5 \mathrm{G}$ and $3 \mathrm{Gcommunications.} \mathrm{It} \mathrm{can} \mathrm{process} \mathrm{motion} \mathrm{videos} \mathrm{and} \mathrm{audios} \mathrm{and} \mathrm{realize} 2 \mathrm{D}$ acceleration, image processing and zoom-in and zoom-out. Integrating MFC, it can support real time video conference and the output of TV signals of NRSC and PAL system. With a built-in most advanced 3D accelerator,

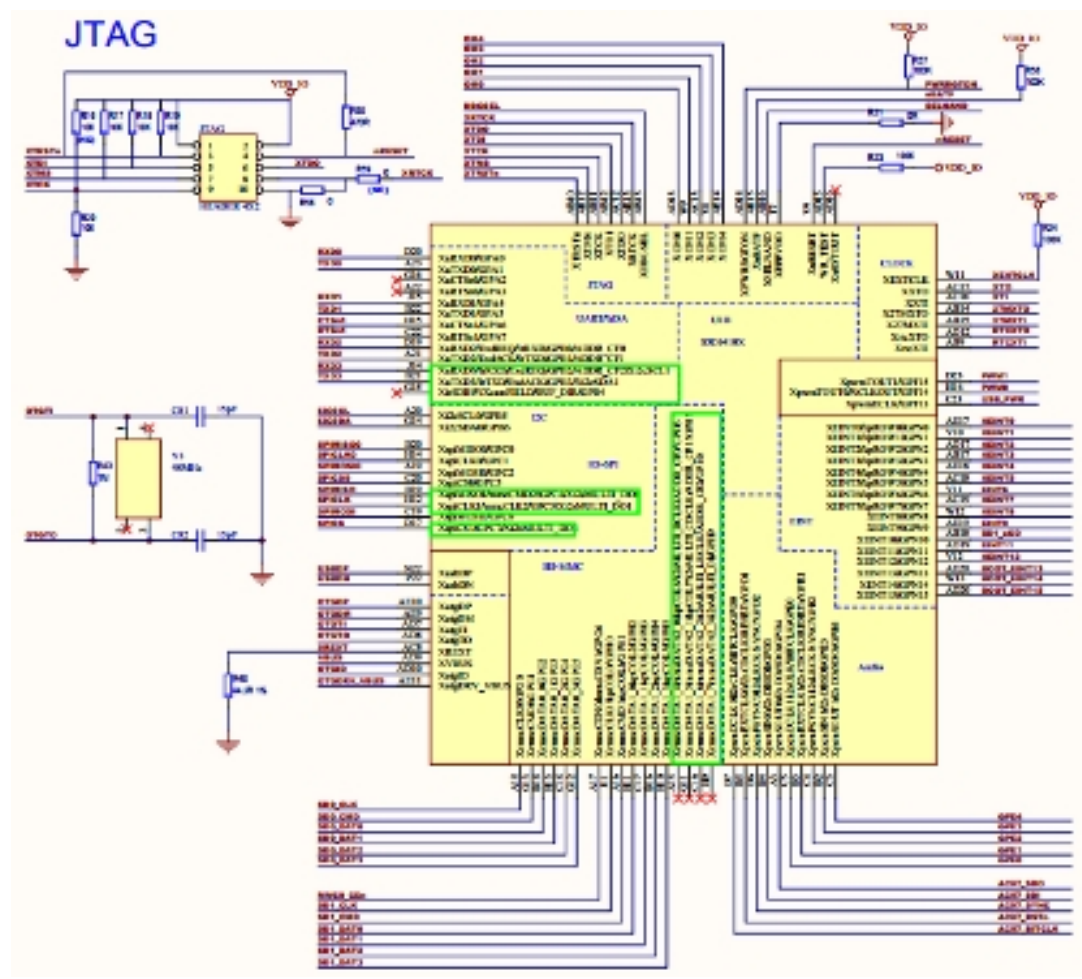

Fig. 5 Motion control processing module 
it supports OpenGL ES1.1/2.0 and D3DM API and can realize the 3D acceleration of 4M triangles/s. The peripheral circuit units, mainly including motor-driven module, ultrasonic distance measuring and sensing module, laser radar module, video capture module and safety test sensor module, are illustrated as the interface in Figure5.

Motor driven circuit This design adopts the DC brush servo motor and IMDR driver for the motor driving module. This module is used for driving three circuits, providing precise closed loop speed control. It also integrates the three-wheel omnidirectional motion control algorithm. With RS232 serial and CAN bus communication, the design realizes motion control of each of the chassis motors. See Fig. 6 for the circuit diagram of the motor driver.

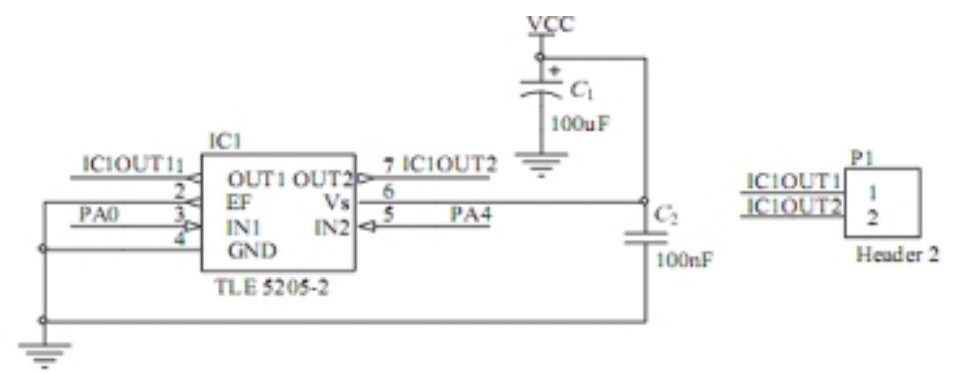

Fig. 6 Circuit diagram of the motor driver

Unit module design for the video capture The video module consists of the steering engine module and the panoramic camera module. According to the design, the digital signals of video data output, filed signals and synchronous clock are connected with S3C6410. See Fig. 7 for the specific connection.

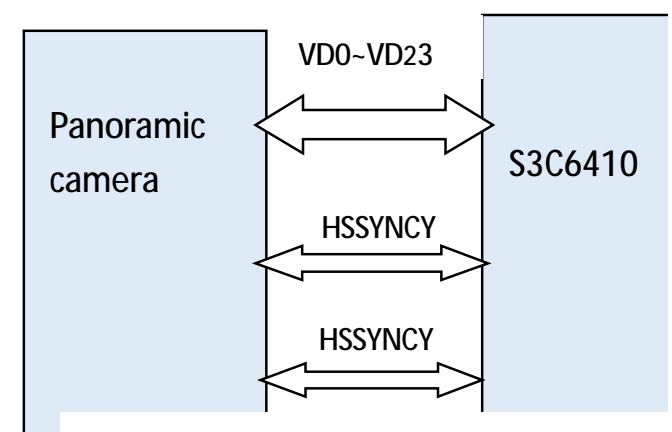

Fig. 7 Video capture module unit

Security detection module unit design The security detection module consists of detection of the indoor temperature and humidity and display, natural gas concentration and alarming, smoke concentration and alarming, and remote smart terminal real-time alarming. See Fig. 8 for the module:

\begin{tabular}{|c|c|c|}
\hline RXD1 & J15 & \\
\hline TXD1 & $\mathrm{B} 22$ & $\begin{array}{l}\text { XuRXDI/GPA4 } \\
\text { XUTYDUGPA5 }\end{array}$ \\
\hline CTSn1 & $\mathrm{H} 15$ & $\begin{array}{l}\text { XuTXDI/GPA } \\
\text { XuCTSDI/GPA }\end{array}$ \\
\hline RTSn1 & $\mathrm{C} 22$ & $\begin{array}{l}\text { XuCTSnI/GPA6 } \\
\text { xuPTSn/GPA7 }\end{array}$ \\
\hline RXD2 & D19 & $\begin{array}{l}\text { XuRTSnl/GPA7 } \\
\text { XuRP?/ExdRF }\end{array}$ \\
\hline TXD2 & $\mathrm{A} 21$ & XuTXD2/ExdACK/IrTXD \\
\hline
\end{tabular}

Fig.8 Infrared security detection module 


\section{Multi-sensor integrated obstacle avoidance module}

The security patrol robot can realize obstacle avoidance. The robot chassis automatically avoid various obstacles in motion with the multi-sense integrated technology ${ }^{[6]}$. The obstacle avoidance and route design can be realized with the information integration of the laser radar, monocular vision and ultrasonic senses. The laser radar obstacle avoidance strategy uses the VHF method ${ }^{[7]}$ to avoid obstacles by using grids to represent the environment and can automatically avoid the obstacles when planning the path. The system receives data from monocular vision panoramic camera and visualizes the data on the visual interface for users to observe and inspect the ambient environment of the robot and "tell" the system to move to the designated position through the interactive entry system. The patrol robot can realize a good self-positioning through its visual sense ${ }^{[8]}$. Its visual system makes navigation through the asterisk visual markings on the ceiling. The robot captures the pictures on ceiling with monocular vision, and extracts the tag characteristics from Opencv library so as to realize the self-location. When the robot enters the roaming status, it will "get lost". At this moment, the identification of the visual tags can allow the robot to rapidly reach the designated position.

The ultrasonic distance sensors are installed on the omnidirectional chassis. Nine ultrasonic distance sensors used based on the shape of the chassis for the long-distance obstacle avoidance, used together with laser radars in coordination. The axis sensing model and the filtering method of tolerance function are used to process this task. The data obtained with these two kinds of sensors will be integrated with the adaptive method. When the distance is far, the ultrasonic data will be given higher weight while when the distance is near and the laser radar data will be given higher weight.

\section{Automatic charging system}

The robot adopts the underneath charging mode with the charge stand. This system drives the robot with eight AA Nickel-cadmium batteries and the machine can reach the charge stand from any direction within the range of $180^{\circ}$. After checking location, the robot starts battery charge and automatically step down the charge stand after completing recharging. It identifies its dynamic environment with the laser radars and visual marking and then plans its path with the fuzzy control to finally realize precise automatic charge.

\section{Smart terminal control unit}

The smart terminal control unit consists of the robot remote control module, monitoring video real-time display module, security indicator display module and security alarm module.

The smart terminal control unit can realize the real-time display of the monitoring video captured by the robot with the monocular vision; real-time display of the security indicators such as temperature, humidity and gas concentration collected by the robot with sensors; remote control of the robot with the remote smart terminal; and realize Internet-based real-time inquiry of voice messages with the voice control platform and LCD interaction based network assistant functions. The user can

Fig.9shows the control of the robot with the buttons on interface of the mobile phone to move forwards, backwards or turn left or right or control the switch of the LED light on eyes of the robot to clearly observe the situation of the house. 


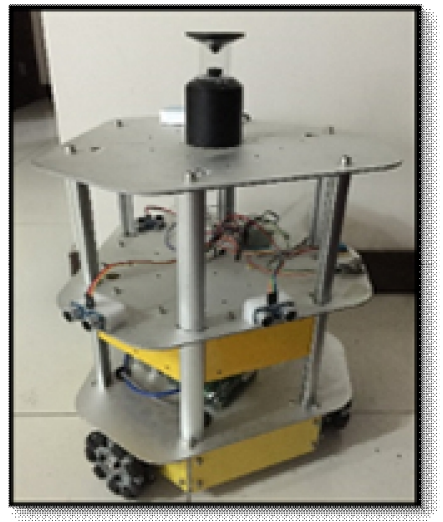

Fig. 9 Mobile phone remote control

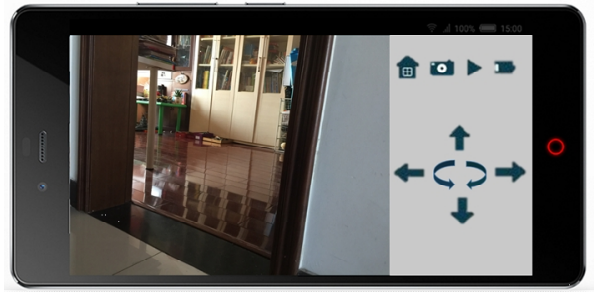

Fig. 10 home patrol robot prototype

\section{Conclusion}

This paper introduces the design of the multi-sensor information system based home security patrol omnidirectional mobile robot. The system adopts the wireless embedded S3C6410 processor and realizes the network remote target image display and control of movement in any direction with the establishment of the three-wheel omnidirectional model; and high-efficient obstacle avoidance with integrated processing of the ultrasonic, laser radar and monocular vision sensors, and monitoring and alarm of daily security of the house with the sensors of gas, smoke and temperatures, and the security patrol robot home entertainment assistant function with the voice interaction module.

\section{References}

[1] Guo Qiaoyun, Xu Xuemei et al. Design of ARM11-based Wireless Video Monitoring System. [J]. Computer Measurement \& Control 2010,18 (8):1786-1787 .

[2] Ma S, Ren C, Ye C. An omnidirectional mobile robot: Concept and analysis[C]//Robotics and Biomimetics (ROBIO), 2012 IEEE International Conference on. IEEE, 2012: 920-925.

[3] Savkin A V, Hoy M. Reactive and the shortest path navigation of a wheeled mobile robot in cluttered environments [J]. Robotica, 2013, 31(02): 323-330.

[4] Ren C, Ma S. Dynamic modeling and analysis of an omnidirectional mobile robot[C]//Intelligent Robots and Systems (IROS), 2013 IEEE/RSJ International Conference on. IEEE, 2013: 4860-4865.

[5] Indiveri G. Swedish wheeled omnidirectional mobile robots: kinematics analysis and control [J]. IEEE transactions on robotics, 2009, 25(1): 164-171.

[6] Wei Wei, Wu Guanjie, Embedded Monocular Robot Visual Inspection and Obstacle Avoidance System Design [J]. Computer Measurement \& Control, 2014.22(11) 3487-3489

[7] Cai zi xing, Zhengmingjie, Zouxiaobing. Real-time obstacle avoidance for mobile robots strategy based on laser radar [J]. Journal of Central South University, 2006,37 (2) : 324-329.

[8] Yu Jinxia, Wang Lu, Cai Zixing, Self-location of Mobile Robot in Unknown Environment [M] . Beijing, Publishing House of the Electronics Industry, 2011 